\title{
Specific glycosylation of $\alpha_{1}$-acid glycoprotein characterises patients with familial Mediterranean fever and obligatory carriers of MEFV
}

\author{
D C W Poland, J P H Drenth, E Rabinovitz, A Livneh, J Bijzet, B van het Hof, W van Dijk
}

\section{Department of Medical Chemistry, Institute for Inflammation and Inflammatory Diseases, Faculty of Medicine, Vrije Universiteit, Amsterdam, The Netherlands D C W Poland $B$ van het Hof W van Dijk}

Department of Medicine, Division of Gastroenterology, University Medical Centre St Radboud, Nijmegen, The Netherlands J P H Drenth

Heller Institute of Medical Research, Sheba Medical Centre, Tel Hashomer and Sackler School of Medicine, Tel Aviv, Israel

E Rabinovitz

A Livneh

Division of Rheumatology, Department of Medicine, University Hospital, Groningen, The Netherlands

\section{J Bijzet}

Correspondence to: Dr W Van Dijk, Department of Medical Chemistry, Institute for Immunology and Inflammatory Diseases, Faculty of Medicine, Vrije Universiteit, Van der Boechorststraat 7, $1081 \mathrm{BT}$ Amsterdam, The

Netherlands

w.van_dijk.medchem @ med.vu.nl

Accepted 23 January 2001

\begin{abstract}
Background-Familial Mediterranean fever (FMF) is a periodic febrile disorder, characterised by fever and serositis. The acute phase response during attacks of FMF results from the release of cytokines, which in turn induce increased expression and changed glycosylation of acute phase proteins. A recent study indicated that attacks in FMF are accompanied by a rise of plasma concentrations of serum amyloid A (SAA) and $C$ reactive protein (CRP), which remain significantly raised during remission relative to healthy controls. Another study suggested that obligatory heterozygotes also display an inflammatory acute phase response.
\end{abstract}

Objective-To determine the state of inflammation in homozygotic and heterozygotic MEFV genotypes.

Methods-CRP and SAA were studied by enzyme linked immunosorbent assay (ELISA). The glycosylation of the acute phase protein, $\alpha_{1}$-acid glycoprotein (AGP), was visualised with crossed affinoimmunoelectrophoresis with concanavalin A as diantennary glycan-specific component and Aleuria aurantia lectin as fucosespecific affinity component.

Results-FMF attacks were associated with an increase $(p<0.05)$ in the serum inflammation parameters CRP, SAA, and AGP. The glycosylation of AGP showed an increase $(p<0.05)$ in fucosylated AGP glycoforms, whereas the branching of the glycans remained unaffected. The glycosylation of AGP in the MEFV carrier group, compared with that in a healthy control group, was characterised by a significant increase $(p<0.05)$ in branching of the glycans, whereas the fucosylation remained unaffected.

Conclusion-The findings suggest an FMF-specific release of cytokines, resulting in a different glycosylation of AGP between a homozygotic and heterozygotic MEFV genotype.

(Ann Rheum Dis 2001;60:777-780)

Familial Mediterranean fever (FMF) is an inherited autosomal recessive inflammatory disease affecting mainly north African Jewish, Armenian, Turkish, and Arab populations. ${ }^{1}$ In more than $80 \%$ of the cases, one or two conservative missense mutations are identified. Most of the mutations are in exon 10 at the carboxy terminal portion of the putative protein encoded by the MEFV gene on chromosome $16 \mathrm{p}^{2}{ }^{3}$ The MEFV gene encodes a protein of 781 amino acids termed pyrin or marenostrin, which is mainly expressed in mature granulocytes and is supposed to play a part in the down regulation of inflammation mediators. ${ }^{2-4}$ Mutations in the MEFV gene are thought to lead to uncontrolled neutrophil activation and migration to serosal tissues. Clinically, FMF is characterised by recurrent attacks of fever, with one or more of the following manifestations: peritonitis, pleuritis, arthritis, myalgia, and erysipelas-like skin lesions. ${ }^{5-7}$ Colchicine is the preferred treatment in FMF and $65 \%$ of patients obtain a complete remission, some $20-30 \%$ show significant improvement with a reduction in the number and severity of attacks, but between 5 and $10 \%$ do not respond to the drug. ${ }^{8}{ }^{9}$ Attacks of FMF are associated with an intense inflammatory response and as a result the acute phase reactants $\mathrm{C}$ reactive protein (CRP), fibrinogen, and serum amyloid A (SAA), the erythrocyte sedimentation rate, and white blood cell count rise sharply. ${ }^{10}{ }^{11} \alpha_{1}$-Acid glycoprotein (AGP), another positive acute phase protein, has not been studied in detail before in FMF.

AGP not only rises during inflammation but also undergoes structural modifications, resulting in a change of both the degree of branching and the extent of $\alpha_{3}$-fucosylation of its glycans. This results in the appearance of a variety of AGP glycoforms in plasma. ${ }^{12-15}$ The change in extent of $\alpha_{3}$-fucosylation is not unique for AGP but has also been described for haptoglobin and $\alpha_{1}$-protease inhibitor. ${ }^{16}$ An increase in $\alpha_{3}$-fucosylation is accompanied by an increased expression of serum blood group determinant sialyl Lewis X. ${ }^{17}$ The state of inflammationthat is, acute or chronic, can be determined by analysing the glycosylation of AGP. During an acute inflammation, as found after an acute insult such as extensive burns or bacterial sepsis, a decrease in branching of the glycans in combination with increased $\alpha_{3}$-fucosylation has been found. ${ }^{12}{ }^{13}{ }^{17-26}$ In contrast, in patients with chronic inflammatory disorders like rheumatoid arthritis or insulin dependent diabetes mellitus, an increased branching with a further increase of $\alpha_{3}$-fucosylation can be found. ${ }^{14} 2728$ Therefore, the changes in relative occurrence of AGP glycoforms, differing in degree of branching and extent of fucosylation, can shed more light on the state of inflammation than the CRP and SAA concentrations alone.

In this study we used the serum concentrations of CRP, SAA, and AGP and the 
Table 1 Concentrations of $a_{1}$-acid glycoprotein (AGP), $C$ reactive protein (CRP), and serum amyloid $A(S A A)$, and reactivities with concanavalin $A$ (Con $A$ ) and Aleuria aurantia lectin ( $A A L)$ of $A G P$ in controls, MEFV carriers, attack and remission. Values are given as means (SD)

\begin{tabular}{lllll}
\hline & Controls $(n=38)$ & MEFV carriers $(n=11)$ & Attack $(n=8)$ & Remission $(n=11)$ \\
\hline AGP $(\mathrm{g} / \mathrm{l})$ & $0.9(0.2)$ & $0.9(0.2)$ & $1.3(0.3)$ & $0.9(0.3)^{\dagger \mathrm{w}}$ \\
CRP (mg/l) & $3.1(3.2)$ & $5.0(9.4)$ & $101.7(110.1)$ & $23.7(51.) 6^{\dagger \mathrm{w}}$ \\
SAA (mg/l) & $1.1(0.8)$ & $6.4(13.7)$ & $214.3(200.8)$ & $54.1(138.5)^{\dagger \mathrm{w}}$ \\
C0 $(\%)$ & $49.9(11.0)$ & $53.2(11.0)$ & $42.7(9.4)$ & $50.4(17.2)$ \\
Cw (\%) & $39.7(6.5)$ & $40.7(7.8)$ & $43.8(4.8)$ & $39.5(9.9)$ \\
Cs (\%) & $10.4(5.2)$ & $6.2(3.9)^{\star \mathrm{t}}$ & $13.5(6.1)$ & $10.1(9.1)$ \\
A0 (\%) & $37.2(15.4)$ & $43.0(24.6)$ & $14.4(6.4)$ & $28.5(17.3)^{\dagger \mathrm{t}}$ \\
Aw+As $(\%)$ & $62.8(15.4)$ & $57.0(24.6)$ & $85.6(6.4)$ & $71.5(17.3)^{\dagger \mathrm{t}}$ \\
\hline
\end{tabular}

* Significantly different from control value $\mathrm{p} \leqslant 0.05$ (two tailed); †significantly different from attack value $\mathrm{p} \leqslant 0.05$ ( 2 -tailed); ${ }^{\mathrm{w}}$ Wilcoxon test; ${ }^{t}$ Student's $t$ test.

$\mathrm{C} 0=\mathrm{Con} \mathrm{A}$ non-reactive fraction; $\mathrm{Cw}=\mathrm{Con} \mathrm{A}$ weakly reactive fraction; $\mathrm{Cs}=\mathrm{Con} \mathrm{A}$ strongly reactive fraction; $\mathrm{A} 0=\mathrm{AAL}$ nonreactive fraction and $\mathrm{Aw}+\mathrm{As}=$ total $\mathrm{AAL}$ reactive fractions.

glycosylation pattern of AGP to investigate the state of inflammation in patients with FMF. Further, we also tested these variables in unaffected MEFV carriers as it was recently suggested that this group had significantly higher CRP and SAA concentrations. ${ }^{10}$

\section{Material and methods}

SOURCE OF SERUM SAMPLES

Serum samples of eight patients with FMF (six male, two female) during attacks, 11 patients with FMF (six male, five female) during remission, $11 \mathrm{MEFV}$ carriers (three male, eight female) were collected for this study. A well defined group, consisting of 38 healthy people with the same ethnic background (nonAshkenazi Jews) and found negative for the three most commonly encountered mutations (M694V, E148Q, V726A) in this group, were used as controls. The mean age of the patients with attacks was 25 years (range 19-38), the mean age of the patients in remission was 30 years (range 20-46), and the mean age of the control group was 38 years (range 18-56). Serum samples were collected from patients seen in the FMF clinic in the Sheba Medical Centre, Tel Hashomer. FMF was diagnosed according to an established set of criteria. ${ }^{29}$

\section{DETERMINATION OF ACUTE PHASE REACTANTS} AGP concentration

Concentrations of AGP were determined by single radial immunodiffusion, according to Mancini et al, using monospecific precipitating anti-AGP antiserum. ${ }^{30}$ Human serum protein calibrator with known AGP concentration, consisting of pooled serum samples from healthy blood donors, was used as a standard for the determination of the AGP concentration.

$C R P$ and $S A A$ concentration

CRP and SAA concentrations were determined by a modification of a previously described enzyme linked immunosorbent assay (ELISA). ${ }^{31}{ }^{32}$ For CRP measurement, goat antihuman CRP, IgG fraction diluted 1:1000 (vol/vol), and biotinylated sheep antihuman CRP ( $1 \mathrm{ng} / \mathrm{ml}$ ) were used as the capture and detection antibody, respectively.

For SAA measurement, the IgG fraction of Reu 86.5 diluted 1:1500 (vol/vol) was used as a capture antibody, and for detection a peroxidase labelled IgG fraction of the monoclonal
anti-SAA Reu 86.1 diluted 1:2000 (vol/vol) was used.

\section{CROSSED AFFINOIMMUNOELECTROPHORESIS}

(CAIE)

This two dimensional electrophoretic technique was performed according to the modified method of Bøg-Hansen. ${ }^{33}$ Two different lectins, concanavalin A (Con A) $(1 \mathrm{mg} / \mathrm{ml})$ (Sigma, St Louis, MO, USA) and Aleuria aurantia lectin (AAL) $(2.5 \mathrm{mg} / \mathrm{ml}$, with a haemagglutination titre of 1/512) (Biomed Labs, New Castle, UK), were used for the binding of diantennary or fucose containing AGP glycoforms. AGP $0.8 \mu \mathrm{g}(1-4 \mu \mathrm{l}$ serum) was electrophoresed through the lectin containing the first dimension gel, and the separated AGP glycoforms underwent immunoelectrophoresis in the second dimension against a precipitating monospecific antiserum. The precipitation curves were visualised by Coomassie brilliant blue R250 staining (Sigma, St Louis, MO, USA). From the areas under the curves the relative amounts of AGP glycoforms were determined by Summagraph (ACECAD D-9000) analysis coupled with an area measurement programme.

\section{STATISTICS}

Results are expressed as mean (SD). Statistical differences between groups were tested by Wilcoxon's test for non-parametrically distributed data and Student's $t$ test for normally distributed data. A two tailed $p$ value of less than 0.05 was considered significant. All calculations were done using SPSS version 9.0.

\section{Results}

CONCENTRATIONS OF AGP, CRP, AND SAA

Table 1 shows that the AGP concentration in the attack group was significantly increased compared with values in the remission group $(\mathrm{p}<0.05)$. The CRP concentration was significant higher during attacks than during remission $(p=0.05)$. The same significant difference was found in SAA concentration between the attack group and the remission group. No significant difference could be detected in AGP, CRP, and SAA concentrations between the control group and the MEFV carrier group.

REACTIVITY OF AGP WITH CON A AND AAL To investigate the glycosylation of AGP with respect to its branching and its $\alpha_{3}$-fucosylation, CAIE was performed with Con A and AAL as 




Figure 1 Crossed affinoimmunoelectrophoresis patterns of $\alpha_{1}$ acid glycoprotein (AGP) with $(A-D)$ concanavalin $A(C o n A)$ and $(E-H)$ Aleuria aurantia lectin $(A A L)$ in serum of a representative control $(A, E), M E F V$ carrier $(B, F)$, patient with familial Mediterranean fever (FMF) during attack $(C, G)$, and a patient with FMF during remission $(D, H)$. The first dimension gel, containing the lectin, was electrophoresed from right to left, the second dimension gel, containing the anti-AGP antiserum, from bottom to top; see "Material and methods" for experimental details. CO and AO = AGP glycoforms that are non-reactive with Con $A$ and $A A L$, respectively; $C w$ and $A w=A G P$ glycoforms that are weakly reactive with Con $A$ and $A A L$, respectively; $C$ s and $A s=A G P$ glycoforms that are strongly reactive with Con $A$ and $A A L$, respectively.

affinity components, respectively. Figure 1 shows CAIE patterns of AGP with Con A and AAL of a representative example of each group. The reactivity of AGP with AAL (Aw+As) was significantly higher in the attack group than in the remission group $(\mathrm{p}<0.05)$, whereas no significant differences in Con A reactivity could be detected (fig 1 and table 1). This suggests that with attacks, the fucosylation of AGP is increased but that the degree of branching remains unchanged. There was a significantly lower Con A reactive fraction (Cs) in the MEFV carrier group than in the control group, indicating a lower degree of diantennary branching of AGP in the MEFV carriers $(p<0.05)$. No significant differences could be shown in AAL reactivity between the two groups (fig 1 and table 1 ).

\section{Discussion}

FMF is characterised by short attacks of fever and serositis associated with an increase of inflammatory mediators such as CRP, SAA..$^{5-7}{ }^{35}$ In this paper we describe the changes in concentration and glycosylation of another acute phase protein, AGP, in patients with FMF during attack and remission, and in carriers of MEFV. Our patients with FMF were clinically diagnosed using rigorous criteria, and in the population studied these criteria have a sensitivity and specificity of $99 \% .{ }^{29}$ In general, genetic analysis of patients with a clinical diagnosis of FMF will yield two mutated alleles in $70 \%$, while in the remaining $30 \%$ only one or even no mutations may be found (data not shown). In the group studied we detected two mutations in 12 patients, one mutated allele in three patients, and none in the remaining four patients (data not shown). Accordingly, we could detect MEFV mutations in seven of the parents of MEFV carriers (data not shown). Our genetic results might be biased because we only searched for three (M694V, V726A, E148Q) mutations. However, these mutations represent the most prevalent MEFV alterations in the studied population. ${ }^{36}$

As indicated by the AAL profiles, an increase in highly fucosylated AGP glycoforms (As), was detected during febrile attacks, whereas in the remission period of FMF the relative occurrence of the highly fucosylated AGP glycoform appears normal. FMF attacks did not influence the degree of branching of the glycans of AGP. The changes in glycosylation of AGP during an attack of FMF point to a hepatic response to chronic inflammation reminiscent of disorders such as rheumatoid arthritis and insulin dependent diabetes mellitus. ${ }^{1427} 28$ The glycosylation pattern of AGP in patients with FMF differs from that of patients with another autosomal recessive inherited periodic febrile disorder: hyper-IgD syndrome (HIDS).$^{37}$ In HIDS we detected an increased $\alpha_{3}$-fucosylation of AGP not only during attacks but also in remission, suggesting a constant chronic hepatic inflammatory response..$^{183} 39$ Furthermore, attacks in HIDS are associated with an increase in diantennary branching of AGP, which returns to normal values in between attacks. Therefore in HIDS, increased $\alpha_{3}$-fucosylation in combination with an increased diantennary glycan content of AGP is evidence for a period of acute inflammation.

The difference in response between both periodic febrile disorders may originate in the completely different profile of cytokine activation during attacks. Cytokines such as interleukin 1 (IL1), IL6, and tumour necrosis factor $\alpha(\mathrm{TNF} \alpha)$ are held responsible for the changed glycosylation of acute phase glycoproteins such as AGP..$^{35}$ In patients with HIDS, a release of IL6, IL1ra, sTNFr p55 and p75, TNF $\alpha$, and interferon $\gamma$ can be found, whereas during an attack of FMF IL1 ra does not rise and appreciable amounts of IL6, sTNFr p55 and p75 only have been detected. ${ }^{35}$

We detected no significant difference in CRP and SAA concentrations in plasma in the MEFV carrier group relative to the healthy control group, which is in contrast with earlier results. ${ }^{10}$ This may be the result of a type 2 error because the concentration of CRP and SAA in plasma in a recently described Dutch control group was shown to be significantly lower than in an MEFV carrier group (data not shown).$^{41}$ Despite similar AGP concentrations in healthy controls and MEFV carriers we found significantly lower amounts of diantennary glycan containing AGP glycoforms in the MEFV carrier group. In contrast, the amount of AGP fucosylation was comparable in both 
groups. This study may indicate that in an MEFV carrier a specific release of cytokines occurs which can modify the branching of AGP but cannot change the fucosylation of AGP.

Research support: Dr J PH Drenth is an investigator of the Royal Netherlands Academy of Sciences and Arts.

1 Daniels M, Shohat T, Brenner-Ulman A, Shohat M. Familial Mediterranean fever: high gene frequency among the non-Ashkenazic Jewish population in Israel. Am J Med Genet 1995;55:311-14

2 The French FMF Consortium. A candidate gene for familial Mediterranean fever. Nature Genetics 1997;17:25-31.

3 The International FMF Consortium. Ancient missense mutations in a new member of the RoRet gene family are likely to cause familial Mediterranean fever. Cell 1997;90: 797-807.

4 Tidow N, Chen X, Muller C, Kawano S, Gombart AF, Fischel-Ghodsian $\mathrm{N}$, et al. Hematopoietic-specific expression of MEFV, the gene mutated in familial Mediterranean fever, and subcellular localization of its corresponding protein pyrin. Blood 2000;4:1451-5.

5 Heller H, Sohar E, Sherf L. Familial Mediterranean fever. Arch Intern Med 1958;102:50-71.

6 Pras M. Familial Mediterranean fever: from the clinical syndrome to the cloning of the pyrin gene. Scand J Rheumatol 1998;27:92-7.

7 Samuels J, Aksentijevich I, Torosyan Y, Centola M, Deng Z, Sood R, et al. Familial Mediterranean fever at the millennium. Clinical spectrum, ancient mutations, and a survey of 100 American referrals to the Nation
of Health. Rev Mol Med 1998;77:268-97.

8 Zemer D, Revach M, Pras M, Modan B, Schor S, Sohar E, et al. A controlled trial of colchicine in preventing attacks of familial Mediterranean fever. N Engl J Med 1974;291: 932-4.

9 Livneh A, Langevitz P, Zemer D, Padeh S, Migdal A, Sohar $\mathrm{E}$, et al. The changing face of familial Mediterranean fever. Semin Arthritis Rheum 1996;26:612-27.

10 Tunca M, Kirkali G, Soyturk M, Akar S, Peyps MB, Hawkins PN. Acute phase response and evolution of familial Mediterranean fever. Lancet 1999;353:1415.

11 Sohar E, Gafni J, Pras M, Heller H. Familial Mediterranean fever. A survey of 470 cases and review of the literature. Am fever. A survey of 470 cas $1967 ; 43: 227-53$.

12 Pos O, Van der Stelt ME, Wolbink GJ, Nijsten MWN, Van der Tempel GL, Van Dijk W. Changes in the serum concentration and glycosylation of alpha1-acid glycoproconcentration and glycosylation of alpha1-acid glycopro-
tein and alpha1-protease inhibitor in severely burned tein and alpha1-protease inhibitor in severely burned patients: relation

13 Mackiewicz A, Marcinkowska-Pieta R, Ballou S, Mackiewicz S, Kushner I. Microheterogeneity of alpha1-acid glycoprotein in the detection of intercurrent infection in systemic lupus erythematosus. Arthritis Rheum 1987;30: 513-18.

14 De Graaf TW, Van Ommen ECR, Van der Stelt ME, Kerstens PJSM, Boerbooms AMTH, Van Dijk W. Effects of low-dose methotrexate therapy on the concentration and glycosylation of alpha1-acid glycoprotein in the serum of rheumatoid arthritis patients: a longitudinal study. J Rheumatol 1994;21:2209-16.

15 Van Dijk W, Brinkman-Van der Linden ECM, Havenaar EC. Glycosyaltion of alpha1-acid glycoprotein (orosomucoid) in health and disease: occurrence, regulation and coid) in health and disease: occurrence, regulation and possible functional implications. Trends in and glycotechnology: TIGG 1998;10:235-45.

16 Brinkman-Van der Linden ECM, Haan PFd, Havenaar EC Van Dijk W. Inflammation-induced expression of sialyl Lewis $\mathrm{x}$ is not restricted to alpha1-acid glycoprotein but also occurs to a lesser extent on alpha1-antichymotrypsin and haptoglobin. Glycoconj J 1998;15:177-82.

17 De Graaf T, Van der Stelt M, Anbergen GM, Van Dijk W. Inflammation-induced expression of sialyl lewis $\mathrm{X}$-containing glycan structures on alpha1-acid glycoprotein (orosomucoid) in human sera. J Exp Med 1993;177: 657-66.

18 Havenaar EC, Drenth JPH, Van Ommen ECR, Van der Meer JWM, Van Dijk W. Elevated serum-level and altered glycosylation of alpha1-acid glycoprotein in hyperimmunoglobulinemia D and periodic fever syndrome: evidence for persistent inflammation. Clin Immunol Immunopathol persistent inflam
19 Nicollet I, Lebreton J-P, Fontaine M, Hiron M. Evidence for alpha1-acid glycoprotein populations of different pI values after concanavalin A affinity chromatography. Study of their evolution during inflam

20 Raynes J. Variations in the relative proportions of microheterogeneous forms of plasma glycoproteins in pregnancy and disease. Biomedicine 1982;36:77-86.

21 Mallet B, Franc JL, Miquel M, Arnaud C. Effects of severe burns on glycan microheterogeneity of four acute-phase proteins. Clin Chim Acta 1987;167:247-57.

22 Pos O, Moshage HJ, Yap SH, Snieders JPM, Aarden L.A., Van Gool J, et al. Effects of monocytic products, recombinant interleukin-1 and recombinant interleukin-6 on the glycosylation of alpha1-acid glycoprotein: studies with primary human hepatocyte cultures and rats. Inflammation 1989;13:415-24.

23 Drechou A, Perez-Gonzalez N, Agneray J, Feger J, Durand G. Increased affinity to concanavalin A and enhanced secretion of alphal-acid glycoproteins by hepatocytes isolated from turpentine-treated rats. Eur J Cell Biol 1989; 50:111-16.

24 Mackiewicz A, Dewey A, Berger FC, Baumann H. Acute-phase mediated change in glycosylation of rat alpha1-acid glycoprotein in transgenic mice. Glycobiology 1991;1:265-9.

25 Turner GA. N-Glycosylation of serum proteins in disease and its investigation using lectins. Clin Chim Acta 1992;208:149-71.

26 Mackiewicz A, Pawlowski T, Mackiewicz-Pawlowska A, Wiktorowicz K, Mackiewicz S. Microheterogeneity forms of alpha1-acid glycoprotein as indicators of rheumatoid arthritis activity. Clin Chim Acta 1987;163:185-90.

27 Havenaar EC, Dolhain RJEM, Turner GA, Goodarzi MT, Van Ommen ECR, Breedveld FC, et al. Do synovial fluid acute phase proteins from patients with rheumatoid arthriacute phase proteins from patients with rheumatoid arth
tis originate from serum? Glycoconj J 1997;14:457-65.

28 Schalkwijk CG, Poland DCW, Van Dijk W, Kok A, Emeis JJ, Dräger AM, et al. Plasma concentration of C-reactive protein is increased in type I diabetic patients without clinical macroangiopathy and correlates with markers of endothelial dysfunction: evidence for chronic inflammation. Diabetologia $1999 ; 42: 351-7$

29 Livneh A, Langevitz P, Zemer D, Kees S, Lidar T, Migdal A, et al. Criteria for the diagnosis of familial Mediterranean fever. Arthritis Rheum 1997;40:1879-85.

30 Mancini G, Carbonara AO, Heremans JF. A single radial diffusion method for the immunological quantification of proteins. Immunochemistry 1965;2:235-54.

31 Hazenberg BPC, Limburg PG, Bijzet J, Van Rijswijk MH. Monoclonal antibody based ELISA for human SAA. VIth International Symposium on Amyloidosis 1990:998-1001.

32 Hazenberg BPC, Limburg PC, Bijzet J, Van Rijswijk MH. SAA versus CRP serum levels in different inflammatory conditions, studied by ELISA using polyclonal anti-AA and monoclonal anti-SAA antibodies. Amyloid and amyloidosis. New York: Plenum Press, 1988:229-33.

33 Bøg-Hansen TC. Crossed immunoaffinoelectrophoresis: an analytical method to predict the result of affinity chromatography. Anal Biochem 1973;56:480-8.

34 Bar-Eli M, Ehrenfeld M, Levy Meal. Leucocyte chemotaxis in recurrent polyserositis (familial Mediterranean fever). Am J Med Sci 1981;281:15-18.

35 Gang N, Drenth JPH, Langevitz P, Zemer D, Brezniak N, Mediterranean fever. J Rheumatol 1999;26:890-7.

36 Livneh A, Langevitz P, Diagnostic and treatment concerns in familial Mediterranean fever. Baillieres Clin Rheumatol 2000;14:477-98.

37 Drenth JPH, Van der Meer JWM. Periodic fevers enter the era of molecular diagnosis. BMJ 2000;320:1091-2.

38 Drenth JPH, Haagsma CJ, Van der Meer JWM, and International Hyper-IgD Study Group. Hyperimmunoglobulinemia $\mathrm{D}$ and periodic fever. The clinical spectrum in a series of 50 patients. Medicine (Baltimore) 1994;73: 133-44.

39 Drenth JPH, Van Deuren M, Van der Ven-Jongekrijg J, Schalkwijk CG, Van der Meer JWM. Cytokine activation
during attacks of the hyperimmunoglobulinemia D and periodic fever syndrome. Blood 1995;85:3586-93.

40 Van Dijk W, Mackiewicz A. Interleukine-6-type cytokineinduced changes in acute phase protein glycosylation. Ann NY Acad Sci 1995;762:319-30.

41 Hazenberg BPC, Limburg PC, Bijzet J, Rijswijk MH. A quantitative method for detecting deposits of amyloid A protein in aspirated fat tissue of patients with arthritis. Ann Rheum Dis 1999;58:96-102. 